\title{
ON A CONJECTURE ON THE CLOSEST NORMAL MATRIX
}

\author{
ANDERS BARRLUND
}

Abstract. Let $A$ be a complex $n \times n$ matrix and let $\mathscr{N}_{n}$ be the set of normal $n \times n$ matrices. A conjecture is that

$$
\left\|A-\mathscr{N}_{n}\right\|_{F}^{2} \leqslant \frac{n-1}{n} \operatorname{dep}^{2}(A),
$$

where $\operatorname{dep}^{2}(A)=\|A\|_{F}^{2}-\sum_{i=1}^{n} \lambda_{i}^{2}(A)$ and $\lambda_{i}(A), i=1, \ldots, n$ are the eigenvalues of $A$. We prove that the conjecture is correct for all even $n$ and for $n=3,5,7$. However, for the dimensions, $n=3,5,6,7$, and presumably also other problem dimensions it is possible to derive sharper bounds. We also prove a bound for odd $n$ which converges to the bound in the conjecture when $n$ tends to infinity. The main idea in the proofs is to use LP problems with constraints based on different ways to approximate $A$ with normal matrices.

Mathematics subject classification (1991): 15A45.

Key words and phrases: Normal matrix, LP-problem.

\section{REFERENCES}

[1] L. Elsner and Kh. D. Ikramov, Towards a proof of László's conjecture, private communication with L. Elsner (hand-written letter from 1996).

[2] P. E. Gill, W. Murray and M. H. Wright, Numerical Linear Algebra and Optimization Vol 1, AddisonWesley, 1990

[3] Kh. D. Ikramov, On normal completions of triangular matrices, Doklady Akademii Nauk, 351 (1996), pp. 1-2 (in Russian).

[4] Kh. D. Ikramov, On normal dilations of triangular matrices, Mathematical notes, 60, N6(1996), pp. 861-872 (in Russian).

[5] L. László, Upper bounds for the best normal approximation, Mathematica Pannonica 9, 1 (1998), pp. $121-129$. 